\title{
Artículo \\ Miedo y Confrontación a la muerte: Estudio en Estudiantes de Ciencias de la Salud
}

\section{Fear and Confrontation to death: Study in Students of Health Sciences}

Autores

Dr. Juan Ignacio Padilla Cuadra, Facultad de Medicina, Universidad de Iberoamérica, UNIBE, San José, Costa Rica

Lic. David Torres Fernandez, Facultad de Psicología, Universidad de Iberoamérica, UNIBE, San José, Costa Rica

MSc. Ericka Coto Jimenez, Facultad de Psicología, Universidad de Iberoamérica, UNIBE, San José, Costa Rica

Dra. Lisette Rodriguez Yebra, Facultad de Farmacia, Universidad de Iberoamérica, UNIBE, San José, Costa Rica MSc. Humberto Fonseca Cordero, Facultad de Enfermería, Universidad de Iberoamérica, UNIBE, San José, Costa Rica Lic. Rosibel Varela Pasos, Unidad de Calidad Academica, Universidad de Iberoamérica, UNIBE, San José, Costa Rica

Correspondencia a: revistamedicina@unibe.ac.cr

\section{Resumen}

La muerte como un evento natural ineludible pero impredecible es un agente generador de ansiedad y temor tanto en pacientes y sus familiares como en profesionales en ciencias de la salud. Es infrecuente que se discuta sobre el tema ni se asignan tópicos en los programas de formación universitaria en ciencias de la salud para el desarrollo de actitudes y competencias especiales en el manejo de pacientes en proceso de morir. Se realizó estudio transversal mediante la aplicación de instrumentos a estudiantes de cuatro carreras de ciencias de la salud para evaluar el nivel de competencia en el afrontamiento según la escala de Bugen, nivel de miedo a la muerte según la escala de
Collett-Lester y se agrega cuestionario para cuantificar el nivel de aceptación a la donación de órganos. Los instrumentos fueron aplicados a un total de 445 estudiantes. La distribución por género fue 97 hombres (21.8\%) y 348 mujeres (78.2 \%). Según facultad, los encuestados quedaron distribuidos en 259 (58.2\%) de medicina, 94 de farmacia (21.1\%), 70 de psicología (15.7\%) y 22 de enfermería ( $5.0 \%$ ). El análisis de confiabilidad de las escalas aplicadas de acuerdo con Alfa de Cronbach fue superior a 0.80. Al analizar los resultados, usando pruebas $\mathrm{T}$ para muestras independientes se encuentran diferencias en religiosidad, miedo a la propia muerte, miedo al propio proceso de muerte, y miedo a la muerte 
de otros. En esto, puntúan más alto las mujeres. No se demostraron diferencias por género en afrontamiento a la muerte o en actitud hacia la donación de órganos. En cuanto a los resultados por facultad, hay diferencias significativas en el miedo al proceso de muerte de otros donde los estudiantes de farmacia reportan puntajes mayores que los de medicina. Además, este mismo grupo de estudiantes (farmacia) reportan menores puntajes en afrontamiento a la muestra que las demás facultades. Se concluye que hay diferencias en cuanto al miedo a la propia muerte entre los estudiantes de diferentes carreras, así como por género. Se recomienda considerar estos resultados en los procesos de diseño curricular para abordar la capacitación en cuanto a actitudes sobre la muerte a estudiantes de ciencias de la salud.

\section{Abstract}

Death as a certain but unpredictable natural event causes anxiety and fear among patients, relatives and healthcare professionals. We carried out a survey through known and validated questionaries to evaluate competence to deal with death (Bugen's score), fear to death score ( Collett-Lester score ) and some additional questions for organ donation acceptance. A total of 445 students were surveyed. The gender distribution was 97 male ( $21.8 \%)$ y 348 females (78.2\%). According to faculty, 259 ( $58.2 \%$ ) were from medicine school, 94 from pharmacy ( $21.1 \%), 70$ from psychology ( $15.7 \%$ ) y 22 from nursing ( $5 \%$ ). Reliability through Alfa coefficient of Cronbach was over 0.80. We found differences on religiosity, fear to death, fear to own process of death and others. On this topic, women had highest scores. There were no differences on gender among coping with death and attitude to organ donation. The results for each faculty showed highest scores on fear to death and lowest scores on coping with death on pharmacy students. We concluded that there are differences on fear to death between students of different career and gender. We recommend these results should be taken on account during curriculum design including training on attitudes toward death upon healthcare sciences students.

\section{Introducción}

La muerte como un evento natural impredecible e inevitable es un agente generador de ansiedad y temor tanto en pacientes, familiares como en profesionales en ciencias de la salud. Estando la sociedad moderna inclinada hacia una actitud de negación hacia la muerte, es infrecuente que se discuta sobre el tema ni se asignen tópicos en los programas de formación universitaria en ciencias de la salud para el desarrollo de actitudes y competencias especiales en el manejo de pacientes en proceso de morir. Por tanto, se considera de interés conocer las actitudes y competencia sobre la muerte en estudiantes de las cuatro carreras en ciencias de la Salud que se imparte en la Universidad de Iberoamérica (San José, Costa Rica). Por todo lo anterior, se propuso realizar la aplicación de dos instrumentos validados especialmente diseñado para tal fin. De igual manera, reconocer la voluntad del entrevistado para la donación de órganos.

\section{Metodología}

Se realizó un estudio transversal mediante la aplicación de instrumentos diseñados y previamente validados para evaluar el nivel de competencia en el afrontamiento según la escala de Bugen, nivel de miedo a la muerte según la escala de Collett-Lester y se incluyeron preguntas para cuantificar el nivel de aceptación a la donación de órganos.

La Escala de Bugen para medir la competencia para afrontamiento ante la muerte cuenta con 30 ítems con una escala de Likert de 1-7 puntos. Por su parte, para medir el nivel de miedo ante la muerte se utilizó la escala de miedo a la muerte de Collett-Lester (CL-FODS por sus siglas en inglés). Este es un instrumento 
multidimensional usado para la evaluación de la ansiedad y el miedo a la muerte que cuenta con cuatro subescalas diferentes: la de miedo a la propia muerte, miedo a la muerte de los demás y las de miedo al proceso de la propia muerte y la de los demás.

Estos instrumentos fueron aplicados directamente a estudiantes de diferentes niveles de las carreras en ciencias de la salud impartidas en esta universidad: medicina, psicología, enfermería y farmacia.

Se incluye en la aplicación de instrumentos Ítems que miden variables demográficas para identificar el perfil de la población: género, edad, carrera y nivel en el programa de estudios

\section{Resultados:}

Los instrumentos fueron aplicados a un total de 445 estudiantes. La distribución por género fue 97 hombres (21.8 \%) y 348 mujeres $(78.2 \%)$. Según facultad, los encuestados quedaron distribuidos en 259 (58.2\%) de medicina, 94 de farmacia (21.1\%), 70 de psicología (15.7\%) y 22 de enfermería (5.0\%).

En cuanto a la distribución por edad, hay un predominio entre 20 a 29 años, seguido por el grupo entre 17 y 19 años.

Gráfico 1. Distribución de Estudiantes de Ciencias de la Salud según Edad.

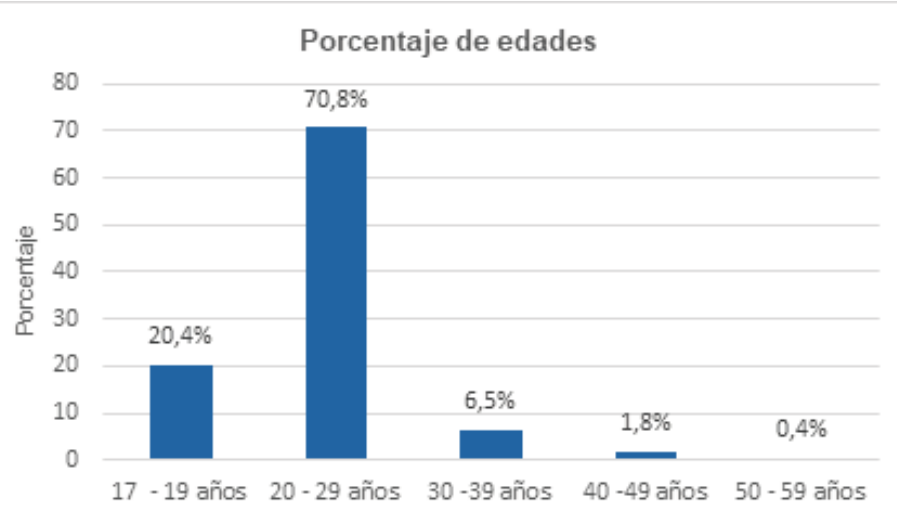

Fuente: Encuesta de Actitudes y Competencias de Afrontamiento ante la Muerte, 2020
Al analizar los resultados por separado de algunas de las preguntas de los diferentes instrumentos aplicados, se demostraron algunas tendencias que se detallan a continuación. Ante la interrogante de si se cuestiona cómo será estar muerto, hay porcentaje considera que indica hacerlo algunas veces y siempre $(58.2 \%)$.

Gráfico 2. Distribución según pregunta ¿Cómo será el estar muerto (a)?

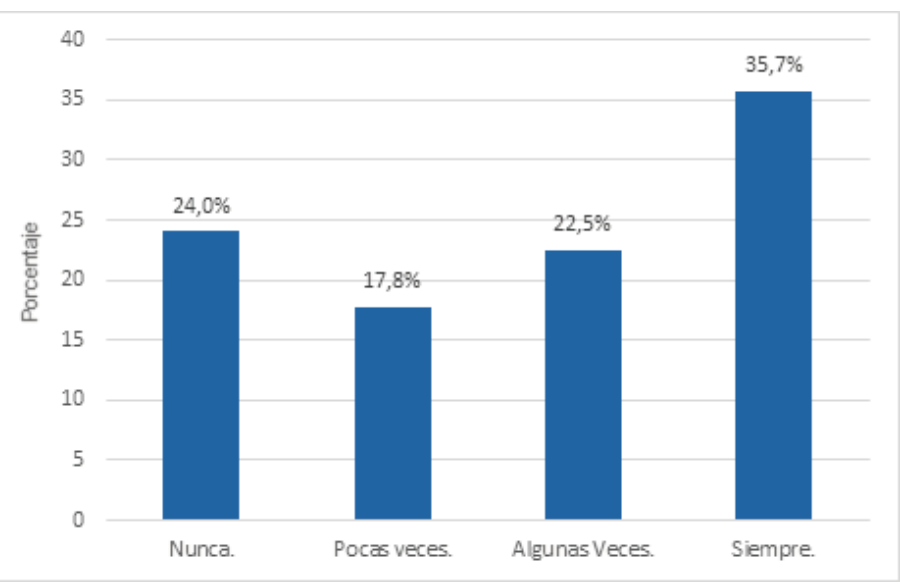

Fuente: Encuesta de Actitudes y Competencias de Afrontamiento ante la Muerte, 2020

Cuando se plantea sobre la mayor preocupación de estar muerto, se muestra que el no poder pensar ni experimentar más no les implica una molestia en especial. 
Gráfico 3. Distribución ante la pregunta de sobre el sentimiento de No poder pensar ni experimentar nada nunca más por la muerte

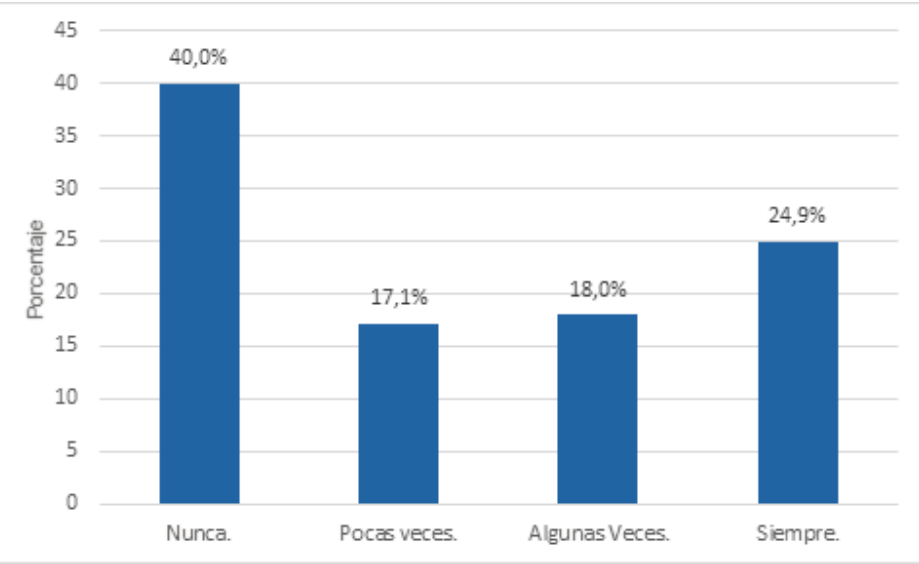

Fuente: Encuesta de Actitudes y Competencias de Afrontamiento ante la Muerte, 2020

Lo mismo cuando se evalúa el temor a la desintegración del cuerpo después de morir, en donde $56.9 \%$ nunca se ha preocupado por eso. No obstante, el morir solo si mostró que es motivo de preocupación o miedo, así como morir joven.

Gráfico 4. Distribución ante la pregunta de sobre el sentimiento de morir solo

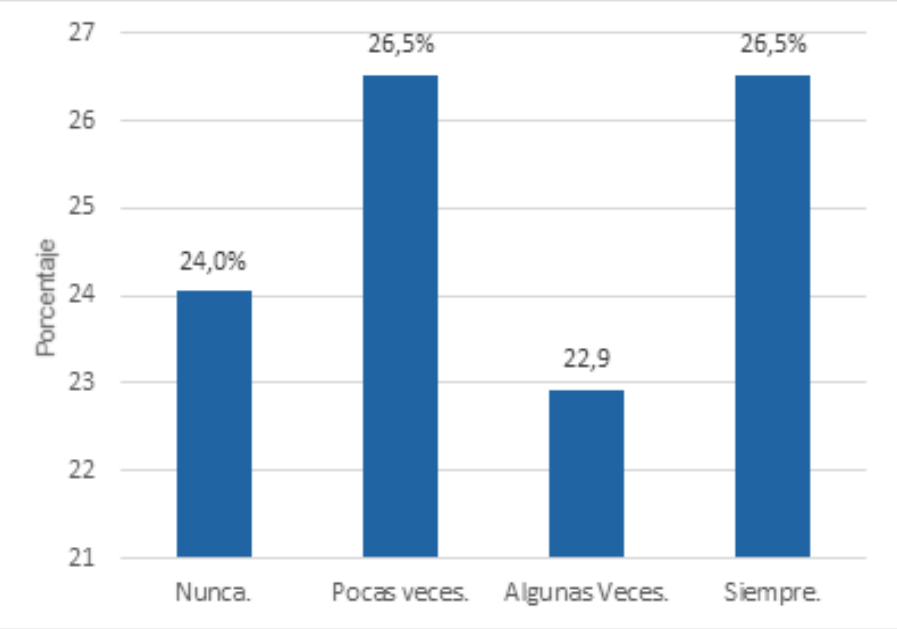

Fuente: Encuesta de Actitudes y Competencias de Afrontamiento ante la Muerte, 2020
Gráfico 5. Distribución ante la pregunta de sobre el sentimiento de morir joven

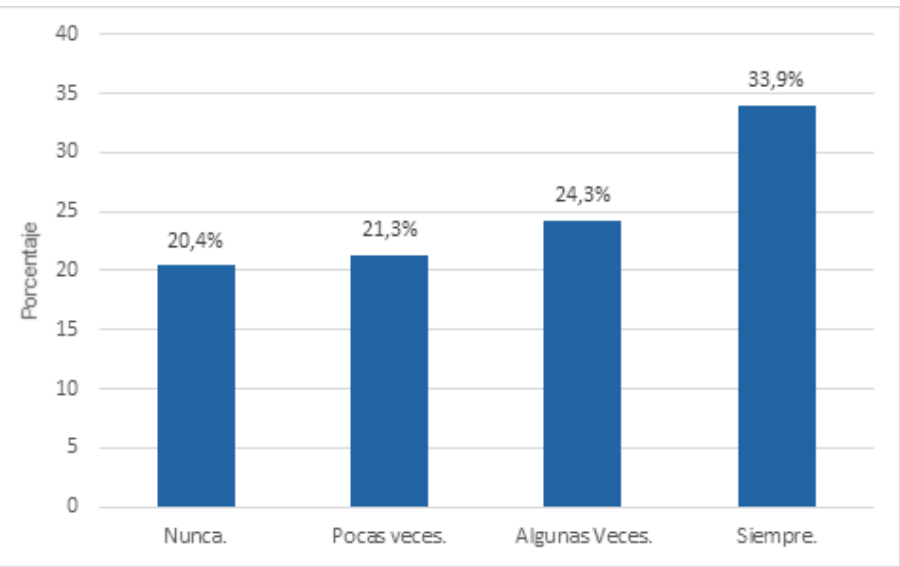

Fuente: Encuesta de Actitudes y Competencias de Afrontamiento ante la Muerte, 2020

La confrontación de la muerte de una persona querida mostró ser motivo de preocupación en un considerable grupo de la población estudiada (siempre: $45.4 \%$ y muchas veces $28.5 \%$ ). Según los resultados estadísticos obtenidos, la razón de dicha preocupación principalmente es no poder comunicarte nunca más con ella (siempre: 45. 2 $\%$ y muchas veces $25.4 \%$ ) y lamentar no haberte llevado mejor con ella cuando aún estaba viva (siempre: $28.3 \%$ y muchas veces $20.4 \%$ )

Debe notarse que, al evaluar la capacidad de confrontar la muerte de una persona querida, no saber cómo gestionar tu dolor ante la pérdida de una persona querida es una preocupación preponderante. 
Gráfico 6. Distribución ante la pregunta de sobre el sentimiento de No saber cómo gestionar tu dolor ante la pérdida de una persona querida

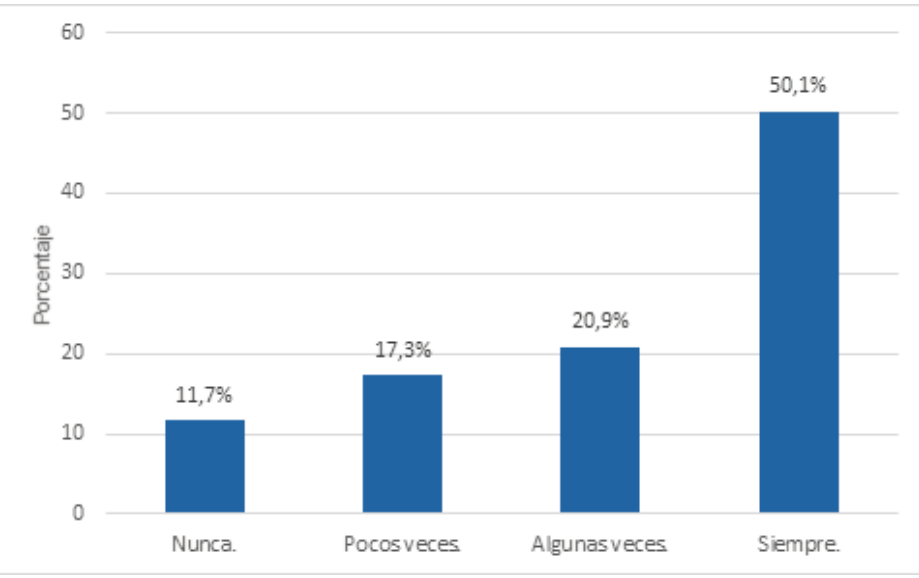

Fuente Encuesta de Actitudes y Competencias de Afrontamiento ante la Muerte, 2020

Es de especial importancia denotar que de la muestra que tiene consciencia que algún día tendrá una experiencia de confrontación con la muerte es relativamente bajo (33.5\%).

Finalmente, mediante el instrumento de aceptación de la donación de órganos se demostró un alto porcentaje.

Gráfico 8. Distribución ante la pregunta de aceptación de la Donación de Órganos

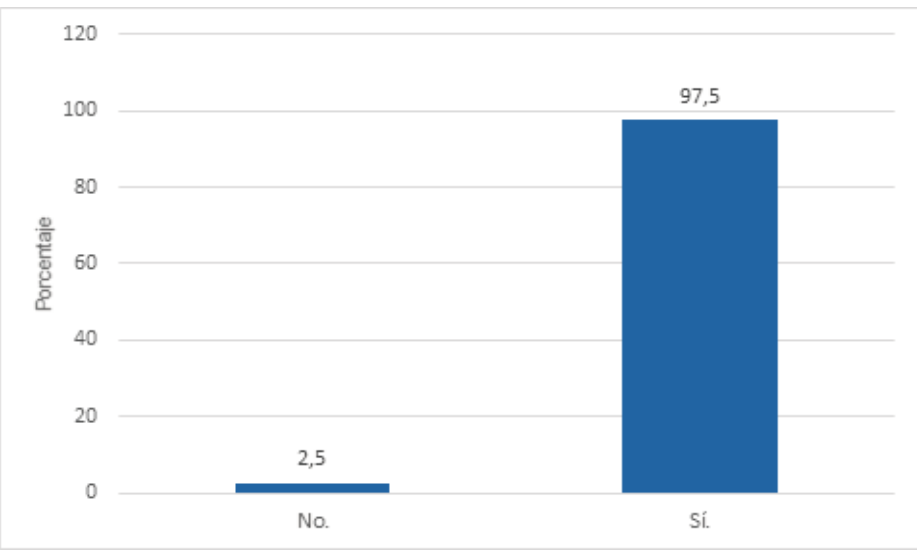

Fuente Encuesta de Actitudes y Competencias de Afrontamiento ante la Muerte, 2020

A lo anterior se muestra una marcada disposición a donar sus órganos luego de morir.
Gráfico 9. Distribución ante la pregunta de aceptación de la Donación de sus Órganos

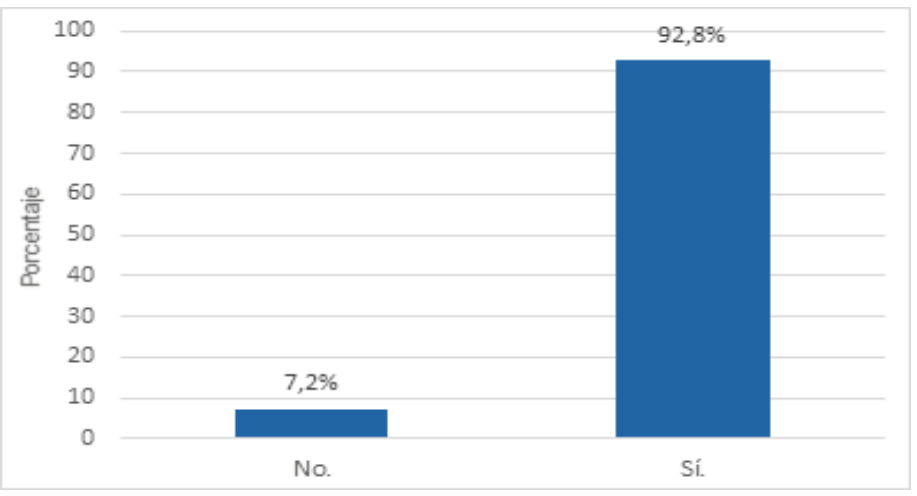

Fuente Encuesta de Actitudes y Competencias de Afrontamiento ante la Muerte, 2020

En cuanto a estos resultados, la confiabilidad de las escalas aplicadas de acuerdo con Alfa de Cronbach fue superior a 0.80 . Al analizar los datos, usando pruebas $\mathrm{T}$ para muestras independientes se encuentran diferencias en religiosidad, miedo a la propia muerte, miedo al propio proceso de muerte, y miedo a la muerte de otros. En todos puntúan más alto las mujeres. No se demostraron diferencias por género en afrontamiento a la muerte o en actitud hacia la donación de órganos.

En cuanto a los resultados por facultad, hay diferencias significativas en el miedo al proceso de muerte de otros donde los estudiantes de farmacia reportan puntajes mayores que los de medicina. Además, este mismo grupo de estudiantes (farmacia) reportan menores puntajes en afrontamiento a la muestre que las demás facultades.

En cuanto a correlaciones entre variables, se encontraron relaciones significativas entre el afrontamiento a la muerte y el miedo a la muerte propio, y al propio proceso de muerte, a la muerte de otros y al proceso de muerte de otros. Siendo a mayor puntaje en afrontamiento a la muerte menor es el miedo. No se encontró relación entre la actitud hacia la donación de órganos con ninguna de la variable medida. 


\section{Discusión}

Debe reconocerse que la muerte y el morir son un estado y proceso generalmente temido (1). Hay aspectos culturales que influyen en la percepción de este proceso. Por ejemplo, en las civilizaciones occidentales, la muerte suele considerarse un tema tabú, limitando una comunicación adecuada en la relación entre el profesional en salud y el paciente (2).

La adecuada atención de pacientes en fase de muerte depende del nivel de capacitación que para tal efecto ha recibido en este campo el profesional en ciencias de la salud. De igual forma existen factores culturales, religiosos y sociales que influyen la actitud con la que los profesionales en salud abordan a los pacientes en esta fase $\mathrm{y}$ a sus familiares. Tales determinantes, en ausencia de una adecuada preparación durante su formación profesional puede conducir a conductas contraproducentes que impiden, no solo al paciente y sus familiares sino también al mismo profesional, asimilar adecuadamente un proceso natural como la muerte.

Al respecto, se han diseñado numerosos instrumentos que buscan definir la actitud y nivel de ansiedad y miedo ante la muerte (3-7). Estos han sido aplicados a diferentes tipos de población incluyendo estudiantes y profesionales en ciencias de la salud, con resultados interesantes (8-11). La importancia de reconocer estas actitudes y el nivel de competencia sobre la muerte en personas que eventualmente tendrán a su cargo este tipo de pacientes radica principalmente en el impacto que esto tenga en:

a. Toma de Decisiones de medidas extremas y limitación de esfuerzos en pacientes críticamente enfermos o en fase terminal

b. Escogencia oportuna de terapia paliativa

c. Propensión a la selección de pacientes potenciales donantes de órganos
La atención de pacientes cuya muerte es inminente e inevitable tiene implicaciones no solo en cuanto a las expectativas de sus familiares, sino también en cuanto al uso de recursos costosos en un entorno con limitaciones. La negación del proceso de muerte como fenómeno natural puede retardar la decisión de escoger medidas paliativas, evitando que la persona enferma reciba una muerte digna provisto de la analgesia necesaria y sin la aplicación de medidas invasivas no solo innecesarias sino fútiles. Finalmente, la tendencia a no reconocer y reclutar potenciales donantes de órganos puede tener relación con el mismo proceso de confrontación ante la muerte por parte de sus médicos tratantes. Este último aspecto es de singular importancia en la actualidad en cuanto diariamente fallecen pacientes por falta de un trasplante siendo una de los factores la cantidad limitada de donantes cadavéricos.

En relación a los instrumentos utilizados es importante identificar algunos aspectos particulares sobre su utilidad. La Escala de Miedo a la Muerte de Collett-Lester es un instrumento multidimensional utilizado para valorar las actitudes hacia la muerte y distingue entre el proceso de muerte propia y la de otros, y ha sido previamente validado en estudios similares (13). Aunque dicha escala se presentó en 1969, nunca fue publicada por sus autores originales, sino que se tiene acceso a la misma por fuentes secundarias. En un estudio realizado por Tomás-Sábado et al, al aplicar la escala de Collett-Lester en 281 estudiantes de enfermería, validaron esta escala y consideran se justifica su uso en profesionales de salud para valorar su actitud hacia la muerte (14). Mondragón-Sánchez et al demostraron al aplicar la escala de Collett-Lester a 641 estudiantes y profesionales en enfermería que el temor a la muerte era menor en estudiantes de primer año con respecto al resto de los entrevistados (15). Este hallazgo puede revelar que la no exposición a situaciones de muerte por 
estar muy temprano en la carrera condiciona este tipo de actitudes. Wood et al demostraron al aplicar la Escala de Collett-Lester que los médicos mostraban menos miedo a la muerte propia y la de otros que los no médicos (16).

Por su parte, la Escala de Bugen, evaluó en un inicio los efectos de la educación en la capacidad para enfrentar el proceso de muerte que trae con consigo repercusiones en la preparación en los profesionales en ciencias de la salud (17). En un estudio similar al presente, Pérez de la Cruz et al, al aplicar la Escala de Bugen a 411 estudiantes de medicina, enfermería y fisioterapia demostraron diferencias significativas entre los tres grupos, mostrando los estudiantes de enfermería el mayor involucramiento emocional en comparación con los estudiantes de las otras carreras. Los autores recomiendan incluir en el currículo de profesionales en salud entrenamiento en el manejo de situaciones al final de la vida (18). Sin embargo, Claxton-Oldfield et al no demostraron que un proceso de entrenamiento variara los puntajes obtenidos en las Escalas de Collett-Lester y Bugen (19).

Una de las principales repercusiones de estos hallazgos, es la interrogante de si la capacitación en este tema podría mejor el nivel de afrontamiento al proceso de muerte en profesionales en salud. Una población profesional especialmente expuesta a la muerte son los dedicados a cuidados paliativos. En este grupo su capacidad para confrontar la muerte ha demostrado un mejor desempeño en su campo (20). La atención adecuada de pacientes en fase de muerte depende del nivel de capacitación que para tal efecto ha recibido en ese campo el profesional en ciencias de la salud. De igual forma existen factores culturales, religiosos y sociales que influyen la actitud con la que los profesionales en salud abordan a los pacientes en esta fase y a sus familiares. Tales determinantes, en ausencia de una adecuada preparación durante su formación profesional puede conducir a abordajes mal orientados que impiden asimilar adecuadamente un proceso natural como la muerte. Esto afecta no solo al paciente, sus familiares sino al profesional e incluso al estudiante. Por tanto, si hay evidencia de que la confrontación ante la muerte causa estrés y dolor en los estudiantes de ciencias de salud y por ende motiva que sea un aspecto a considerar durante su formación (21) (22).

En cuanto a la actitud sobre la donación de órganos en estudiantes de ciencias de la salud, Padilla et al reportaron al aplicar encuestas a 326 estudiantes ciencias de la salud, que un 90,8\% mostró disposición a donar sus órganos (23), similar a lo demostrado en el presente estudio. Al considerar en el actual estudio, si el nivel de competencia al afrontar la muerte y el miedo a la muerte, podían condicionar la aceptación a la donación de órganos, no se demostró relación alguna con los resultados de los instrumentos aplicados.

Según estudios previos, debe mencionarse que a mayor grado socioeconómico y la educación mayor la aceptación a la donación de órganos (24). Dicha actitud puede variarse de manera positiva mediante la introducción de programa educativos sobre el tema (25).

En conclusión, la aplicación de los instrumentos escogidos demostró que hay diferencias en cuanto al miedo a la propia muerte entre los estudiantes de diferentes carreras, así como por género. El análisis individual de algunas preguntas demuestra preocupación a la confrontación del proceso de muerte de otros. Hay aceptación considerable a la donación de órganos. Se recomienda considerar estos resultados en los procesos de diseño curricular para abordar la capacitación en cuanto a actitudes sobre la muerte a estudiantes de ciencias de la salud. Se propone que con esto se podría mejorar su habilidad para confrontar este proceso. Es importante que exista 
conciencia sobre el proceso de muerte, en tanto, por la naturaleza de su carrera, habrá alta probabilidad de exposición a estas situaciones.

\section{Referencias}

1. Benbunan Bentata B, Cruz Quintana F, Roa Venegas JM, Bettina C, Benbunan Bentata R. Afrontamiento del dolor y la muerte en estudiantes de enfermería: una propuesta de intervención. Int J Clin Health PsychoI 2007; 7:197-205.

2. Cardozo R, Sosa M, Gómez A, Sánchez A, Sosa G, Bastidas G, Guevara H, Ortunio M. Apreciaciones sobre la muerte en estudiantes del último año de medicina. VITAE 2010; 11 (44): disponible en:

http://vitae.ucv.ve/pdfsNITAE_4263.pdf.

3. Neimeyer RA, Wittkowski J, Moser RP. Psychological research on death attitudes: an overview and evaluation. Death Stud. 2004 May;28(4):309-40.

4. Collett, L. J., \& Lester, D. The fear of death and the fear of dying. Journal of Psychology 1969; 72:179-181.

5. Robbins, R. A. Bugen's Coping with Death Scale: Reliability and further validation. Omega 1991;22, 287-299.

6. Robbins, R. A. Death competency: A study of hospice volunteers. Death Studies 1992; 16: 557-569.

7. Robbins, R. A. Death competency: Bugen's Coping with Death Scale and Death Self-Efficacy. In R. A. Neimeyer (Ed.), Death anxiety handbook (pp. 149-165). New York: Taylor \& Francis. 1994

8. Sábado T, Limonero J, y Abdel-Khalek A. Spanish adaptation of the Collett Lester fear of death scale. Rev Death Stud. 2007;31-60.
9. Edo-Gual M, Tómas - Sábado J, Aradilla Herrero A. Miedo a la muerte en estudiantes de enfermería. Enferm Clin $2011 ; 21$ :129-35.

10. Espinoza Venegas M, Sanhueza Alvarado O, Barriga O. Validación de la Escala de Miedo a la Muerte de Collett-Lester en una muestra de estudiantes de Enfermería Rev. Latino-Am. Enfermagem 2011; 19(5):1-10.

11. Galiana L, et al. Validación Confirmatoria de la Escala de Afrontamiento de la Muerte en Profesionales de Cuidados Paliativos 2015; 181: 1-10.

12. Firestone, R. W. (1994). Psychological defenses against death anxiety. In R. A. Neimeyer (Ed.), Death anxiety handbook: Research, instrumentation, and application (pp. 217-241). New York: Taylor \& Francis.

13. Lester, David, The Collett-Lester fear of death scale: the original and a revision. Death Studies 1990; 14: 451-468.

14. Tomás-Sábado J, Limonero J, Abdel- Khalek A. Spanish Adaptation of the Collett-Lester Fear of Death Scale, Death Studies 2007; 31:3, 249-260.

15. Mondragón-Sánchez E, Torre Cordero E, Morales Espinoza M, Landeros-Olvera E. A comparison of the level of fear of death among students and nursing professionals in Mexico Rev. Latino-Am. Enfermagem 2015 Mar.-Apr.;23(2):323-8

16. Wood K, Robinson PJ. Fear of death in a sample of physicians. Can Fam Physician. 1984 Feb;30:416-20.

17. Bugen, L. A., Coping: Effects of Death Education, Omega, 11:2, pp. 175-183,1980-81.

18. Pérez- de la Cruz S, García- Luengo AV. Comparative study among Spanish students of health sciences degrees: Facing death. Nurs Health Sci. 2018;1-7. 
19. Claxton-Oldfield S, Crain M, Claxton-Oldfield J. Death anxiety and death competency: the impact of a palliative care volunteer training program. Am J Hosp Palliat Care. 2006 Dec-2007 Jan;23(6):464-8.

20. Schmidt-RioValle J, Campos-Calderon CP, Garcia-Caro MP, Prados-Peña D, CruzQuintana F. Efectos de un programa de formación en cuidados paliativos sobre el afrontamiento de la muerte. Med Paliat. 2012;19(3):113-20.

21. Corr, C.A. Death in modern society. In Oxford Texbook of Palliative Medicine; Doyle, D., Hanks, G.W.C.Y.,MacDonald, N., Eds.; Oxford Medical Publications: Oxford, UK, 1983; pp. 28-36.

22. Tomás Sábado J, GuixLlistuella, E. Ansiedad ante la muerte: Efectos de un curso de formación en enfermeras y auxiliaries de enfermería. Enfermería Clínica 2001, 11, 104-109.

23. Padilla Cuadra JI, Mora Chacón P, Monge Fallas A, Rodríguez barquero R. Actitudes y conocimientos sobre la donación de órganos, trasplante y muerte cerebral en estudiantes de ciencias de la salud. Acta Méd Costarricense 2015: 57(4): 180-183.

24. Saleem T, Ishaque S, Habib N, Hussain SS, Jawed A, Khan AA, Ahmad MI, Iftikhar MO, Mughal HP, Jehan I. Knowledge, attitudes and practices survey on organ donation among a selected adult population of Pakistan. BMC Med Ethics. 2009;10:5.

25. McGlade D, Pierscionek B. Can education alter attitudes, behaviour and knowledge about organ donation? A pretest-post-test study. BMJ Open 2013;3: e003961. 